\title{
Un caso di epatite colestatica da ticlopidina: descrizione del caso e revisione della letteratura
}

\author{
Ticlopidine-induced cholestatic hepatitis: A case report and review \\ of the literature
}

\section{Luigi Anastasio ${ }^{a, *}$, Valerio Manno ${ }^{a}$, Maria Carbone ${ }^{a}$, Nicola Lentini ${ }^{a}$, Sandro Baldari ${ }^{\mathrm{b}}$, Lucia Sofia ${ }^{\mathrm{a}}$, Giuseppe Topa ${ }^{\mathrm{a}}$, Antonio Arone ${ }^{\mathrm{a}}$}

\author{
${ }^{a}$ UOC Medicina Interna (Direttore: Dott. Luigi Anastasio), Ospedale G. Jazzolino, Vibo Valentia \\ ${ }^{\mathrm{b}}$ UO Radiodiagnostica (Direttore: Dott. Sandro Baldari), Ospedale G. Jazzolino, Vibo Valentia
}

Ricevuto il 22 giugno 2011; accettato il 21 ottobre 2011

disponibile online il 3 aprile 2012

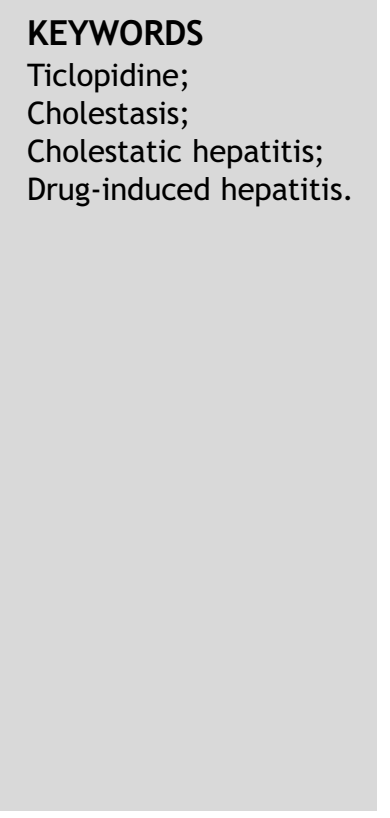

\begin{abstract}
Summary
Introduction: Cholestatic hepatitis is frequently a drug-related syndrome. We describe the case of a 57-year-old man who developed cholestatic hepatitis two months after starting therapy with ticlopidine following a carotid endarterectomy.

Materials and methods: The patient presented with anorexia, nausea, and dark-colored urine. The work-up included laboratory tests and imaging studies of the liver (ultrasound and magnetic resonance imaging). The authors analyze the case using the scale developed by Maria and Victorino for the diagnosis of drug-induced hepatitis, the Naranjo algorithm for adverse drug reactions, and the RUCAM algorithm for causality assessment of hepatotoxicity. They also review data from the MedLine database on cases of ticlopidine-induced cholestatic hepatitis reported during the period 1982-2011.

Results: Bilirubin, aminotransferases, alkaline phosphatases, and gamma glutamyl transpeptidase levels were elevated at admission and progressively declined after ticlopidine was discontinued. The absence of biliary obstruction at ultrasonography and magnetic resonance cholangiography, the negative results of viral and immunologic tests, and the resolution of the syndrome after discontinuation of the drug all suggested ticlopidine-induced hepatotoxicity. The assessment of this case with toxicity algorithms confirmed that a causal link to ticlopidine was "probable" or "highly probable." The patient was treated with ursodesoxycholic acid, clopidogrel $(75 \mathrm{mg} /$ day), and (after the laboratory parameters had normalized) rosuvastatin $(10 \mathrm{mg} /$ day). No further clinical and laboratory abnormalities have been observed during two month follow-up.
\end{abstract}

\footnotetext{
* Corrispondenza: via Aversa Precenzano 14 - 88046 Lamezia Terme (CZ).

E-mail: luigi.anastasio@aliceposta.it (L. Anastasio).
} 
Discussion: The toxicity of ticlopidine is well established: our review revealed reports of 57 cases of ticlopidine-induced cholestatic hepatitis during the period 1982-2011. The mechanisms underlying the toxic effects of this drug are not clear, but they are probably related to the chemical structure of the drug. The syndrome is usually completely reversible with discontinuation of the drug. We stress the importance for the appropriate use of this drug and the need for adequate follow-up of patients.

(c) 2012 Elsevier Srl. All rights reserved.

\section{Caso clinico}

Il paziente, maschio di 57 anni, era giunto alla nostra osservazione per una sintomatologia insorta circa 7 giorni prima e caratterizzata da inappetenza, dispepsia, nausea, emissione di urine di colore scuro. All'atto del ricovero il paziente presentava ittero, epatomegalia, lieve splenomegalia.

L'anamnesi remota del paziente poneva in evidenza un intervento chirurgico inguinale, per motivi non precisati (ernioplastica?), in età infantile. Nessuna patologia degna di nota fino a 3 anni prima del ricovero, epoca nella quale, per il riscontro di elevati valori pressori, il paziente iniziava una terapia antipertensiva. Nello stesso periodo il paziente veniva sottoposto a colecistectomia per una calcolosi biliare. Un'ecografia, eseguita 2 anni prima del ricovero per motivi non precisati, evidenziava una modesta epato-splenomegalia non approfondita successivamente.

Due mesi prima del ricovero il paziente veniva sottoposto, per il riscontro di una stenosi carotidea severa, a tromboendoarteriectomia chirurgica. Dopo l'intervento il paziente iniziava trattamento con ticlopidina, ancora in corso al momento del ricovero, alla dose di $250 \mathrm{mg} 2$ volte al di per os. Oltre alla ticlopidina, al momento del ricovero il paziente assumeva anche $10 \mathrm{mg} /$ die di rosuvastatina (da circa $6 \mathrm{mesi}$ ), $20 \mathrm{mg} /$ die di olmesartan (da circa 2 anni), $20 \mathrm{mg} /$ die di lercanidipina (da oltre 1 anno). Egli dichiarava di essere stato fumatore, fino a 2 mesi circa prima del ricovero, di 25 sigarette al giorno per oltre 30 anni. Inoltre, lo stesso era bevitore di circa mezzo di litro di vino ai pasti. Il paziente, di mestiere muratore, negava l'uso di tossici professionali nel corso della sua attività lavorativa e l'assunzione di sostanze o farmaci a scopo ricreativo o di automedicazione (in particolare paracetamolo).

All'ingresso in reparto il paziente presentava un'obiettività clinica caratterizzata da: sensorio integro con normali condizioni di vigilanza e cognizione; cute idratata, di colorito diffusamente itterico; masse muscolari toniche e trofiche; obesità di grado lieve (indice di massa corporea 31); decubito indifferente. L'esame del collo evidenziava una cicatrice chirurgica a destra, da pregressa endoarteriectomia carotidea. Non si rilevavano deficit neurologici sensitivo-motori. L'esame del cuore e del torace era nella norma. Il fegato era palpabile a circa $6 \mathrm{~cm}$ dall'arcata costale e di normale consistenza. Era palpabile il polo inferiore della milza.

L'elettrocardiogramma evidenziava ritmo sinusale a frequenza cardiaca $65 \mathrm{bpm}$, tratto PR $0,16 \mathrm{~s}$, asse elettrico deviato a sinistra intorno $\mathrm{a}-15^{\circ}$, non anomalie significative della ripolarizzazione. L'ecocardiogramma non evidenziava elementi di rilievo, tranne un modesto aumento dell'ecogenicità dei lembi mitralici con rigurgito non significativo della valvola. Normale la pressione polmonare stimata mediante analisi del rigurgito tricuspidale.
Gli esami di laboratorio mettevano in evidenza alterata glicemia a digiuno e normalità della funzione renale, degli elettroliti, dell'esame emocromocitometrico, degli indici comuni di coagulazione. Si riscontrava, invece, un notevole incremento degli indici di danno epatico (AST 312; ALT 137; GGT 819) e degli indici di colestasi (bilirubina totale 8,2; bilirubina diretta 8,1 ; bilirubina indiretta 0,1 ; fosfatasi alcalina 429). L'andamento di queste variabili è riportato nelle figura 1 e 2. Nei giorni successivi si è osservato un ulteriore incremento dei valori delle aminotransferasi, più marcato per l'ALT, cui è seguito un progressivo declino, molto più rapido per l'AST, che nell'arco di 60 giorni è ritornata alla normalità.

L'ecografia addominale, eseguita in seconda giornata, poneva in evidenza un fegato di dimensioni aumentate, a ecostruttura finemente disomogenea come per epatopatia diffusa. Vie biliari di normale calibro. Vena porta di $13 \mathrm{~mm}$. Splenomegalia (diametro longitudinale $181 \mathrm{~mm}$ ).

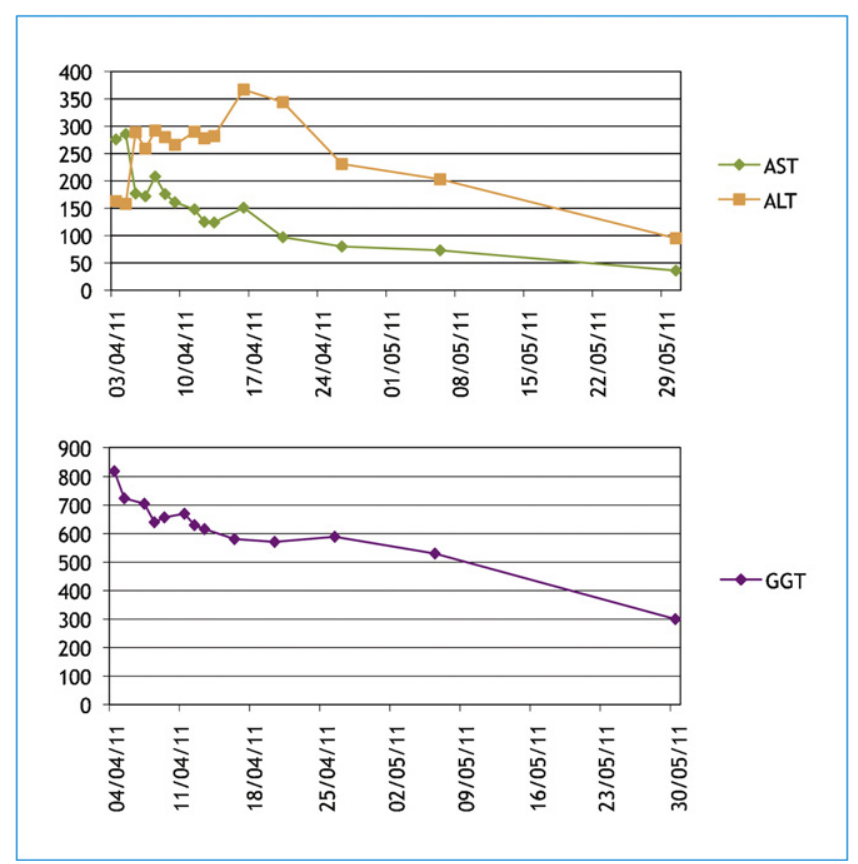

Figura 1 Andamento temporale dei valori relativi alle aminotransferasi (AST e ALT) e alla gamma-glutamil-transpeptidasi (GGT). I valori di AST rientrano nella norma nell'arco di circa 60 giorni, mentre quelli di ALT persistono elevati (2,3 volte il limite superiore della norma) a 60 giorni, sia pure con un andamento in riduzione parallelo a quello di AST. La GGT va incontro a una progressiva riduzione dei valori, persistendo tuttavia valori pari a circa 6 volte il limite della norma ancora a 60 giorni dalla sospensione della ticlopidina. 

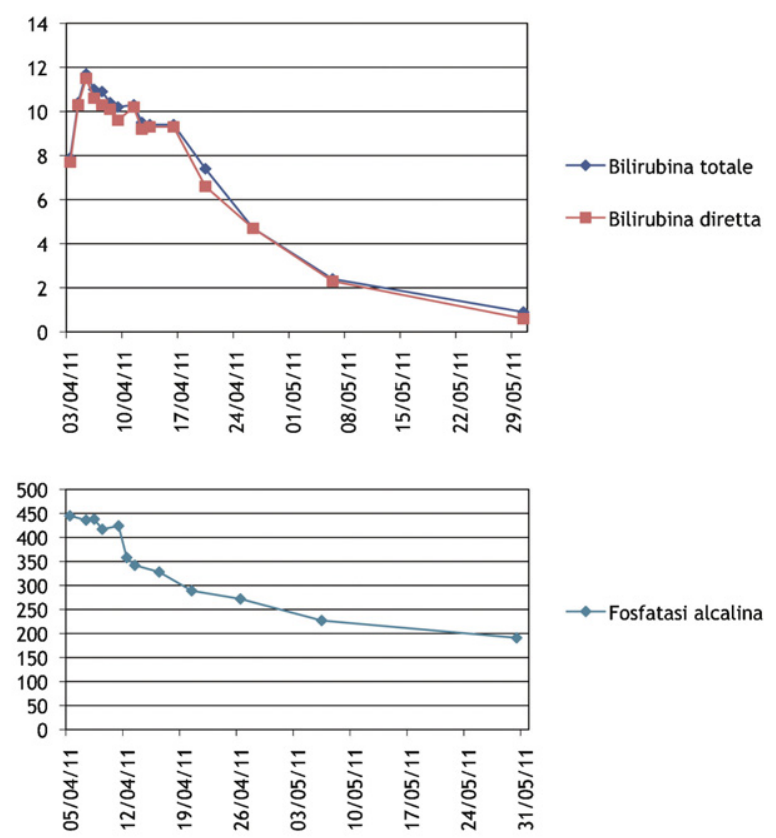

Figura 2 Andamento temporale dei valori relativi alla bilirubina totale e coniugata e alla fosfatasi alcalina. La normalizzazione dei valori della bilirubina si è osservata nell'arco di circa 60 giorni. La fosfatasi alcalina è andata incontro a un sollecito dimezzamento dei valori, per poi diminuire linearmente fino a valori prossimi alla normalità nell'arco di circa 60 giorni.

La TC dell'addome superiore, eseguita senza e con contrasto, esame mirato allo studio del pancreas, evidenziava un pancreas di dimensioni nei limiti a densità parenchimale omogenea. Non evidenza di versamento libero in addome. Vie biliari non dilatate. Esiti di colecistectomia. Vena porta pervia e di calibro regolare. Splenomegalia $(180 \mathrm{~mm})$.

La RM dell'addome superiore, senza e con contrasto, mirata allo studio delle vie biliari, dimostrava la presenza di vie biliari regolari per intensità di segnale e calibro ed esiti di colecistectomia.

La negatività degli esami ematochimici e sierologici relativi alle ipotesi di epatopatia autoimmune, virale, cirrosi biliare, nonché la negatività della colangio-RM, eseguita alla ricerca di un disturbo di canalizzazione delle vie biliari correlato alla colangite sclerosante, ha orientato il percorso diagnostico verso la possibilità di una causa farmacologia. In effetti, l'interruzione dell'assunzione di ticlopidina da parte del paziente, contestuale al ricovero, ha consentito di osservare un progressivo, ma evidente, declino degli indici di colestasi, con una cronologia variabile per i diversi indici (figg. 1 e 2).

L'applicazione al caso descritto della scala di Maria e Victorino [1], utilizzata ormai da oltre un decennio per la valutazione del grado di plausibilità di attribuzione a un farmaco di una reazione tossica rilevata, ha prodotto un punteggio di $14 / 20$, che fa rientrare il caso nella categoria "probabile". È stato omesso il "rechallenge test", che avrebbe consentito di raggiungere la certezza di attribuzione della reazione alla ticlopidina, in quanto ritenuto non etico. D'altra parte la scala di Naranjo et al. [2], ideata per testare l'attribuzione all'uso di farmaci di un fenomeno clinico, applicata al nostro caso, fornisce un punteggio di $7 / 13$, che qualifica la tossicità da ticlopidina anche in questo ambito come “probabile”. L'applicazione dell'algoritmo conosciuto con l'acronimo RUCAM [3,4], riferito specificamente alle reazioni di tossicità epatica, evidenzia un punteggio di 9/15, che colloca il ruolo della ticlopidina, come causa della reazione epatotossica nel nostro caso, nella categoria "altamente probabile".

Durante la degenza il paziente ha sospeso l'assunzione di ticlopidina e rosuvastatina e ha assunto $40 \mathrm{mg} /$ die di prednisone per 10 giorni. Il paziente è stato dimesso in decima giornata, in buone condizioni generali, asintomatico, con un programma di follow-up ambulatoriale fino a normalizzazione degli indici più importanti di colestasi, proseguendo il trattamento antiaggregante con clopidogrel $75 \mathrm{mg}$ e la terapia epatoprotettiva con acido ursodesossicolico $300 \mathrm{mg}$ 3 volte al dì. Non è stato ripreso il trattamento con statina fino a normalizzazione delle transaminasi. La successiva ripresa dell'assunzione di rosuvastatina non ha dato luogo ad alterazioni degli indici biochimici nei successivi 2 mesi di follow-up.

\section{Discussione}

La ticlopidina [5-(2-chlorofenil)metil-4,5,6,7-tetraidrotieno[3,2-c] piridina] (fig. 3) è un potente inibitore dell'aggregazione piastrinica indotta dall'adenosin-difosfato (ADP) mediante l'inibizione dell'attivazione del complesso formato dal recettore per l'ADP e la glicoproteina di membrana IIb/IIla. L'effetto antiaggregante si ottiene entro 24-48 ore dalla somministrazione per via orale, mentre l'effetto massimo si realizza entro 3-6 giorni dalla prima dose e persiste fino a 72 ore dall'ultima assunzione [5]. L'assorbimento intestinale è all'incirca dell'80-90\%, mentre il metabolismo è epatico, con almeno un metabolita attivo. L'eliminazione avviene per il $60 \%$ a livello urinario e per il $25 \%$ circa con le feci. La ticlopidina è un inibitore a spettro piuttosto ampio del citocromo P450, anche se, a concentrazioni clinicamente rilevanti, inibisce il P450 2C19 umano e il 2D6.

Sebbene efficace nella prevenzione di eventi aterotrombotici a livello cardio e cerebrovascolare, nonché vascolare periferico, l'assunzione di ticlopidina è risultata determinare una significativa incidenza di eventi tossici a livello ematologico [6,7], quali agranulocitosi, porpora trombotica trombocitopenica, e anemia aplastica. È stata inoltre segnalata

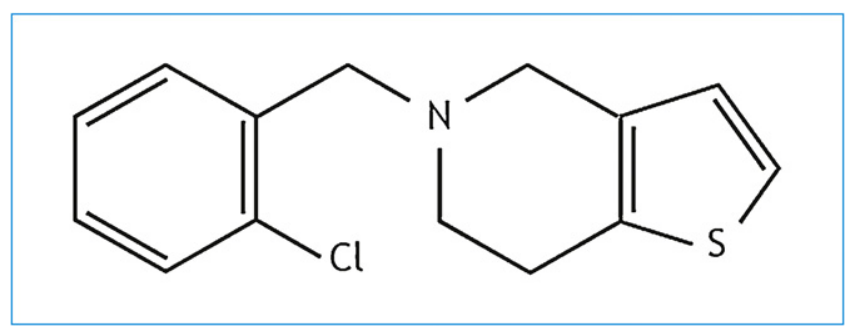

Figura 3 Formula di struttura della ticlopidina. 
una significativa tossicità epatica, caratterizzata da incremento, anche asintomatico, delle aminotransferasi.

La complicanza di cui qui si tratta, legata all'utilizzo della ticlopidina e caratterizzata da una sindrome colestatica intraepatica, è stata segnalata sin dal 1982 [8] e finora in letteratura sono stati pubblicati almeno 57 casi [9-59]. La sindrome colestatica, stando a quanto rilevato dall'esame dei casi descritti, solitamente regredisce, purché riconosciuta, con la sospensione del farmaco. Contrariamente a quanto notato per altri farmaci capaci di indurre danno colestatico, che nel $10 \%$ dei casi è un danno persistente, non sono stati finora descritti casi di cronicizzazione della sindrome colestatica da ticlopidina, anche se le alterazioni biochimiche possono persistere fino a oltre un anno dopo la sospensione del farmaco [31,38,40]. Più frequentemente, i sintomi e le anomalie biochimiche si risolvono nell'arco di 1-3 mesi.

La severità della colestasi è notevolmente variabile, con valori di bilirubina ampiamente oscillanti da 0,7 fino a 26 mg/dL (media 9,6 mg \%). La fosfatasi alcalina può aumentare fino a 5-10 volte $i$ valori normali e le aminotransferasi presentano un ampio spettro di valori (41-2661 UI/L, con una media di $425 \mathrm{UI} / \mathrm{L})$.

L'unico caso mortale sinora descritto non è dovuto all'evoluzione del quadro verso l'insufficienza epatica, dovendosi attribuire il decesso alla presenza di un'agranulocitosi complicata da uno stato settico [45].

Il periodo di latenza, compreso fra l'inizio del trattamento con ticlopidina e l'insorgenza di segni o sintomi di tossicità epatica, è variabile nella letteratura fra una settimana e 6 mesi [32], essendo comunque nella maggior parte dei casi compreso fra 2 e 12 settimane. In un caso descritto in letteratura, l'insorgenza del quadro colestatico si è verificata addirittura un mese dopo la sospensione del farmaco, motivata invece dall'insorgenza di un rash cutaneo [37].

Poiché la maggior parte dei pazienti affetti da epatite colestatica indotta da ticlopidina sono anziani (età media 67,5 anni; range 29-92 anni), alcuni autori hanno ipotizzato una maggiore suscettibilità a questa complicanza in età avanzata [46], anche se tale reperto appare influenzato, piuttosto, dalla particolare composizione della popolazione trattata con il farmaco.

Poche sono le biopsie epatiche eseguite, a quanto riferito dalla letteratura, sia per l'età generalmente avanzata dei soggetti sia per l'evoluzione benigna della condizione morbosa. L'istologia generalmente ha dimostrato la presenza di colestasi o di un aspetto misto di colestasi e danno epatocellulare. Sono stati evidenziati anche infiltrati di mononucleati o misti, mononucleati e neutrofili, con aspetti granulomatosi, a livello degli spazi portali [31,37-39]. La biopsia, ripetuta dopo la risoluzione clinica in un singolo caso, ha dimostrato la piena risoluzione del quadro epatico microscopico [49].

Il meccanismo mediante il quale la ticlopidina è in grado di indurre il danno epatico colestatico non è noto. Dall'esame della letteratura non sembra emergere una problematica dose-dipendente: anzi, nel 20\% dei casi descritti il farmaco era somministrato a dosi subterapeutiche $(250 \mathrm{mg} / \mathrm{die}$ in unica somministrazione). Sono prospettati sia effetti mediati dal sistema immunitario, con ipersensibilità al farmaco, sia effetti tossici diretti della ticlopidina o dei suoi metaboliti [43].
Uno studio [47] ha mostrato un'anomala stimolazione, da parte della ticlopidina, dei linfociti $T$ di soggetti affetti da epatopatia colestatica ticlopidino-indotta, evidenziata attraverso l'incorporazione di timidina tritiata in vitro da parte delle cellule $\mathrm{T}$, rispetto ai linfociti provenienti da soggetti di controllo. La presenza, nel $40 \%$ circa dei casi descritti in letteratura (ma non nel caso da noi presentato), di manifestazioni di ipersensibilità (febbre, esantema cutaneo, eosinofilia) suggerisce la possibilità di un meccanismo immunoallergico. Allo stesso modo, il rilievo delle citate lesioni granulomatose alla biopsia epatica, in almeno due casi della letteratura, sostiene questa ipotesi [60,61].

Tuttavia è ipotizzabile anche un meccanismo metabolico, basato sul riscontro della possibilità di indurre una colestasi sperimentale mediante infusione di boli ripetuti di $10 \mathrm{mg}$ di ticlopidina [18]. La ticlopidina, infatti, è metabolizzata dal fegato mediante processi di $\mathrm{N}$-dealchilazione, $\mathrm{N}$-ossidazione e ossidazione dell'anello tiofenico a quattro diversi metaboliti. Differenze dettate dalla diversa velocità individuale delle vie metaboliche, o dalla differente espressione nei singoli delle stesse, potrebbero essere alla base di possibili effetti idiosincrasici [37]. Il potenziale epatotossico potrebbe essere anche in relazione con la presenza nella molecola della ticlopidina dell'anello tiofenico, in analogia con altri composti capaci di indurre epatotossicità come l'anello tienilico, presente in alcune tienodiazepine, quali il clotiazepam e il bentazepam [62].

L'utilizzo dei corticosteroidi nel trattamento della colestasi indotta da ticlopidina non è codificato: potrebbe avere un ruolo nell'agevolare la guarigione, in analogia con quanto avviene in altri casi di epatocolestasi indotta da farmaci $[52,63]$. Alla luce della scarsa documentazione di efficacia, dell'abituale benignità della condizione e del rischio non trascurabile di eventi avversi, l'uso degli steroidi dovrebbe essere riservato ai casi nei quali, sulla base di una valutazione individualizzata, se ne possa ipotizzare l'utilità.

L'acido ursodesossicolico è stato impiegato in qualche caso sulla base del suo profilo di sicurezza e tollerabilità, alla luce della sua capacità di limitare l'espressione degli antigeni HLA di classe I negli epatociti e di ridurre il numero di linfociti T attivati [47].

La possibilità di sostituire la ticlopidina che abbia indotto danno colestatico con il clopidogrel, nonostante l'affinità strutturale delle due molecole (fig. 4), deriva dal fatto che

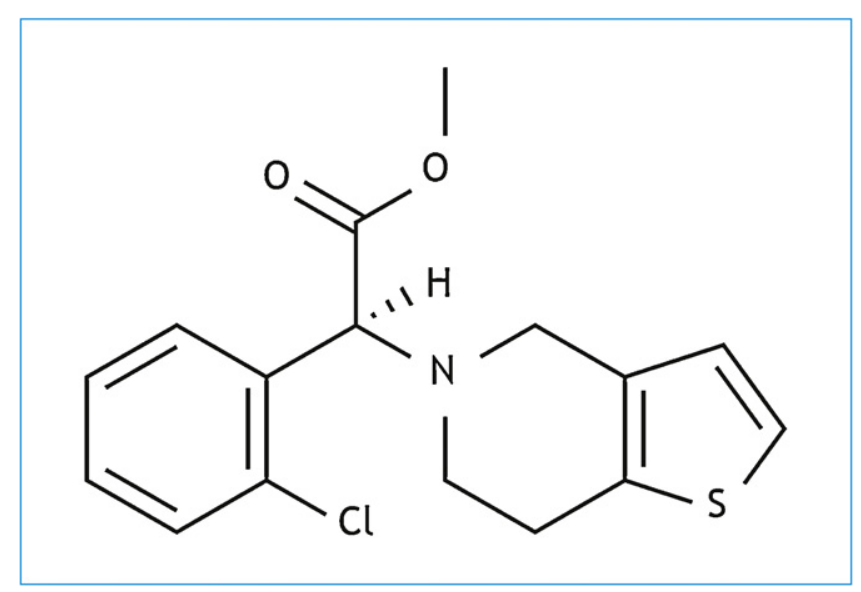

Figura 4 Formula di struttura del clopidogrel. 
quest'ultimo farmaco risulta molto meno capace di indurre la colestasi e, in alcuni casi riportati in letteratura, è stato utilizzato con successo come antiaggregante in pazienti affetti da colestasi da ticlopidina [59,64].

In una serie di casi di epatotossicità segnalati in letteratura [10] risulta che il 30\% circa dei pazienti ha ricevuto il farmaco per un'indicazione non approvata e il $20 \%$ circa a dosi non adeguate; cifre analoghe (rispettivamente, $48 \%$ di inappropriatezza e $32 \%$ di non corretto dosaggio) sono riportate in un'altra serie [65]. La potenziale tossicità della ticlopidina deve indurre i medici a un uso oculato e soprattutto appropriato di questo farmaco, effettuando una sorveglianza clinica e di laboratorio prolungata anche oltre quanto attualmente suggerito dalla schede tecniche.

\section{Conflitto di interessi}

Gli autori dichiarano di non aver nessun conflitto di interessi.

\section{Bibliografia}

[1] Maria VA, Victorino RM. Development and validation of a clinical scale for the diagnosis of drug-induced hepatitis. Hepatology 1997;26(3):664-9.

[2] Naranjo CA, Busto U, Sellers EM, Sandor P, Ruiz I, Roberts EA, et al. A method for estimating the probability of adverse drug reactions. Clin Pharmacol Ther 1981;30(2):239-45.

[3] Danan G, Benichou C. Causality assessment of adverse reactions to drugs-I. A novel method based on the conclusions of international consensus meetings: application to drug-induced liver injuries. J Clin Epidemiol 1993;46(11):1323-30.

[4] Benichou C, Danan G, Flahault A. Causality assessment of adverse reactions to drugs-II. An original model for validation of drug causality assessment methods: case reports with positive rechallenge. J Clin Epidemiol 1993;46(11):1331-6.

[5] Saltiel E, Ward A. Ticlopidine. A review of its pharmacodynamic and pharmacokinetic properties, and therapeutic efficacy in platelet-dependent disease states. Drugs 1987;34(2):222-62.

[6] Lesesve JF, Callat MP, Lenormand B, Monconduit M, Noblet C, Moore $\mathrm{N}$, et al. Hematological toxicity of ticlopidine. Am J Hematol 1994;47(2):149-50.

[7] Love BB, Biller J, Gent M. Adverse haematological effects of ticlopidine. Prevention, recognition and management. Drug Saf 1998;19(2):89-98.

[8] Deschamps JP, Lassègue $A$, Ottignon $Y$, Vuitton $D$, Allemand $H$, Carayon $\mathrm{P}$, et al. Cholestatic jaundice associated with ingestion of ticlopidine: a propos of the first 2 cases. Gastroenterol Clin Biol 1982;6(6-7):595-6.

[9] Saint-Marc Girardin MF, Cordonnier C. Cholestatic icterus and agranulocytosis due to ticlopidine. Gastroenterol Clin Biol 1982;6(8-9):716-7.

[10] Eugene C, Lefebvre JF, Gury B, Quevauvilliers J. Cholestatic hepatitis. Presumptive role of ticlopidine. Sem Hop 1983; 59(42):2923-4.

[11] Pistone AM, Podestà F, Raviolo E, Testa D, Grosso B, Toselli P, et al. Cholestatic fatty liver of probably iatrogenic origin. Recenti Prog Med 1986;77(4):188-90.

[12] Mammarella A, Paoletti V, Moroni C, Cassone R. Ticlopidineinduced cholestatic jaundice. Clin Ter 1991;138(1):45-6.

[13] Greany Jr JJ, Hess DA, Mahoney CD. Ticlopidine-induced cholestatic jaundice. Clin Pharm 1993;12(6):398-9.

[14] Nurhussein MA. Ticlopidine-induced prolonged cholestasis. J Am Geriatr Soc 1993;41(12):1371-2.
[15] Sondag D, Bader R, Claude P, Schreiber M. Hepatitis due to ticlopidine: a new case. Ann Gastroenterol Hepatol (Paris) 1993;29(1):40-1.

[16] Elizaga Corrales J, Martín Herráez A, Reverte Cejudo D. Cholestatic jaundice secondary to ticlopidine. An Med Interna 1993;10(12):621-2.

[17] Grimm IS, Litynski JJ. Severe cholestasis associated with ticlopidine. Am J Gastroenterol 1994;89(2):279-80.

[18] Álvaro D, Gigliozzi A, Gaudio E, Pescarmona E, Ripani M, Peri C, et al. Ticlopidine-induced cholestasis: reports of two cases and experimental evidence of the drug's adverse effect in the isolated perfused rat liver. Eur J Gastroen Hepat 1994;6:943-50.

[19] Weber E, Donckier J. Cholestatic jaundice due to ticlopidine: a new case. Acta Clin Belg 1994;49(6):309-10.

[20] Yoder JD, Algozzine GJ, Hill GW. More ticlopidine-induced cholestatic jaundice. Am J Hosp Pharm 1994;51(14):1821-2.

[21] Colivicchi F, Magnanimi S, Sebastian F, Silvestri R, Magnanimi R. Ticlopidine-induced chronic cholestatic hepatitis: a case report. Curr Ther Res 1994;55:929-31.

[22] Naschitz JE, Khamessi R, Elias N, Yeshurun D. Ticlopidine-induced prolonged cholestasis. J Toxicol Clin Toxicol 1995;33(4): $379-80$.

[23] Roy L, Plante MA, Perreault H, Biron P. Cholestatic hepatitis: ticlopidine suspected. Therapie 1995;50(6):593-4.

[24] Cassidy LJ, Schuster BG, Halparin LS. Probable ticlopidineinduced cholestatic hepatitis. Ann Pharmacother 1995;29(1): $30-2$.

[25] López P, Castiella A, Bujanda L, Arenas Jl. Ticlopidine-induced cholestatic hepatitis. A case report. Rev Esp Enferm Dig 1995;87(10):735-7.

[26] Miras Parra FJ, Gómez Jiménez FJ, García Contreras T, Valverde Romera M. Ticlopidine-induced cholestatic hepatitis. Rev Esp Enferm Dig 1995;87(5):414-5.

[27] Pascual S, Sarrión JV, Jarque I, Argüello L, Berenguer J. Cholestatic hepatitis and anemia induced by ticlopidine. Gastroenterol Hepatol 1996;19(4):208-9.

[28] Díaz Fuenzalida A, Valdés Socín H, Laudano O, Avagnina A, Findor JA. Cholestasis associated with ticlopidine. Gastroenterol Hepatol 1997;20(3):128-30.

[29] Sánchez-Bisonó JR, Gómez-Moli J, Escudero-Cantó M. Probable ticlopidine-induced severe aplastic anemia and cholestatic hepatitis. Haematologica 1997;82(5):639.

[30] Guzzini F, Banfi L, Gomitoni A, Marchegiani C, Novati P, Mesina $M$, et al. Two cases of acute cholestasis caused by ticlopidine. Recenti Prog Med 1997;88(3):124-7.

[31] Iqbal M, Goenka P, Young MF, Thomas E, Borthwick TR. Ticlopidine-induced cholestatic hepatitis: report of three cases and review of the literature. Dig Dis Sci 1998;43(10):2223-6.

[32] Grieco A, Vecchio FM, Greco AV, Gasbarrini G. Cholestatic hepatitis due to ticlopidine: clinical and histological recovery after drug withdrawal. Case report and review of the literature. Eur J Gastroenterol Hepatol 1998;10(8):713-5.

[33] Wu MS, Chan P, Lien GS, Cheng YS, Pan S. Ticlopidine-induced severe cholestatic hepatitis. Zhonghua Yi Xue Za Zhi (Taipei) 2000;63(8):663-6.

[34] Berent R, Hinterholzer G, Höbling W, Auer J, Haidenthaler A, Knoflach $P$. Cholestatic hepatitis as a rare side effect of therapy with ticlopidine. Z Gastroenterol 2000;38(7):587-91.

[35] de la Iglesia Martínez F, Trinidad Sanjosé JC, Nicolás Miguel R, Ramos Polledo V, Pellicer Vázquez C, Diz-Lois Martínez F. Ticlopidine induced cholestatic hepatitis. An Med Interna 2000;17(9):504-5.

[36] García Ortega P, Navarro JJ, Carvajal A, García del Pozo J. Cholestatic hepatitis by ticlopidin. Med Clin (Barc) 2001; 116(3):117.

[37] Kubin CJ, Sherman O, Hussain KB, Feinman L. Delayed-onset ticlopidine-induced cholestatic jaundice. Pharmacotherapy 1999;19(8):1006-10. 
[38] Meyer MI, Kuhn M, Bühler H, Bertschinger P. Ticlopidine-induced cholestasis. Schweiz Med Wochenschr 1999;129(39):1405-9.

[39] Wegmann C, Münzenmaier R, Dormann AJ, Huchzermeyer $H$. Ticlopidine-induced acute cholestatic hepatitis. Dtsch Med Wochenschr 1998;123(6):146-50.

[40] Amaro P, Nunes A, Maçôas F, Ministro P, Baranda J, Cipriano A, et al. Ticlopidine-induced prolonged cholestasis: a case report. Eur J Gastroenterol Hepatol 1999;11(6):673-6.

[41] Torrano Larrión F. Cholestatic hepatitis from ticlopidine. Aten Primaria 1999;24(2):115.

[42] San Juan Portugal F, Jiménez Saez J, Naya Manchado J, Fuentes Solsona F. Cholestatic hepatitis due to ticlopidine: a report of a new case. An Med Interna 1997;14(10):540-1.

[43] Bruguera M. Cholestatic hepatitis associated with ticlopidine. Gastroenterol Hepatol 1997;20(3):163-4.

[44] Pizarro AE, Andrade RJ, García-Cortés M, Lucena MI, PérezMoreno JM, Puertas M, et al. Acute hepatitis due to ticlopidine. A report of 12 cases and review of the literature. Rev Neurol 2001;33(11):1014-20.

[45] Ceylan C, Kirimli O, Akarsu M, Undar B, Güneri S. Early ticlopidine-induced hepatic dysfunction, dermatitis and irreversible aplastic anemia after coronary artery stenting. Am J Hematol 1998;59(3):260.

[46] Klepser TB, Jogerst GJ. Ticlopidine-induced elevated liver enzymes. Pharmacotherapy 1997;17(4):819-21.

[47] Skurnik YD, Tcherniak A, Edlan K, Sthoeger Z. Ticlopidineinduced cholestatic hepatitis. Ann Pharmacother 2003;37(3): $371-5$.

[48] Tsai MH, Tsai SL, Chen TC, Liaw YF. Ticlopidine-induced cholestatic hepatitis with anti-nuclear antibody in serum. J Formos Med Assoc 2000;99(11):866-9.

[49] Chen LK, Hsieh BH, Chen WC, Tsai ST, Hou MC. Ticlopidineinduced hepatitis. Zhonghua Yi Xue Za Zhi (Taipei) 2001;64(1): 59-63.

[50] Tsui PT, Lai ST, Leung WS, Mok NS, Wu CW, Lau ST, et al. Prolonged jaundice following percutaneous coronary intervention and ticlopidine therapy. Hong Kong Med J 2002;8(1):57-9.

[51] Rivera Vaquerizo P, Solís García del Pozo J, Villanueva Hernández P. Ticlopidine-induced hepatitis. Report of a new case. An Med Interna 2002;19(2):99-100.

[52] Alberti L, Alberti-Flor JJ. Ticlopidine-induced cholestatic hepatitis successfully treated with corticosteroids. Am J Gastroenterol 2002;97(4):1072.
[53] Adams L, Jeffrey GP, Deboer B, Garas G. Ticlopidine-associated cholestatic hepatitis. Intern Med J 2002;32(7):359-60.

[54] Yamamoto N, Shiraki K, Saitou Y, Kawakita T, Okano H, Sugimoto $\mathrm{K}$, et al. Ticlopidine induced acute cholestatic hepatitis complicated with pure red cell aplasia. J Clin Gastroenterol 2004;38(1):84.

[55] Gandolfi A, Mengoli M, Rota E, Tolomelli S, Zanghieri G, Bernini MV, et al. Ticlopidine-induced acute cholestatic hepatitis. A case report. Recenti Prog Med 2004;95(2):96-9.

[56] Mambelli E, Mancini E, Casanova S, Di Felice A, Santoro A. Severe ticlopidine-induced cholestatic syndrome. Blood Purif 2007;25(5-6):441-5.

[57] Lee JY, Park EB, Ahn JH, Suh SJ, Jung YK, Kim JH, et al. A case of ticlopidine induced acute cholestatic hepatitis and pure red cell aplasia. Korean J Hepatol 2008;14(1):102-7.

[58] Kowalski R, Dropiński J, Brzostek T, Szot P, Rzeszutko M, Kasper $M$, et al. Cholestatic hepatitis as a ticlopidine-induced complication of treatment-A case report. Kardiol Pol 2008; 66(7):758-60.

[59] Anselmino M, Moretti C, Ravera L, Sheiban I. Clopidogrel treatment in a patient with ticlopidine-induced hepatitis following percutaneous coronary stenting. Minerva Cardioangiol 2010; 58(2):277-80.

[60] Ruiz-Valverde P, Zafon C, Segarra A, Ribera R, Piera L. Ticlopidine-induced granulomatous hepatitis. Ann Pharmacother 1995;29(6):633-4.

[61] Flamenbaum $M$, Zenut $M$, Castillo $D$, Costes-Charlet $N$, Kemeny JL, Lavarenne J, et al. Granulomatous hepatitis and ticlopidine. Therapie 1997;52(6):610-1.

[62] Andrade RJ, Lucena MI, Aguilar J, Lazo MD, Camargo R, Moreno $\mathrm{P}$, et al. Chronic liver injury related to use of bentazepam: an unusual instance of benzodiazepine hepatotoxicity. Dig Dis Sci 2000;45(7):1400-4.

[63] Goland S, Malnick SD, Gratz R, Feldberg E, Geltner D, Sthoeger ZM. Severe cholestatic hepatitis following cloxacillin treatment. Postgrad Med J 1998;74(867):59-60.

[64] Zeolla MM, Carson JJ. Successful use of clopidogrel for cerebrovascular accident in a patient with suspected ticlopidine-induced hepatotoxicity. Ann Pharmacother 1999;33(9): 939-41.

[65] del Pino AM, de Abajo FJ, Montero D, Madurga M, MartínSerrano G. Ticlopidine use in Spain: a pharmacy-based drug utilization study. Med Clin (Barc) 2000;115(6):211-3. 